\title{
Quitline Use and Outcomes among Callers with and without Mental Health Conditions: A 7-Month Follow-Up Evaluation in Three States
}

\author{
Katrina A. Vickerman, ${ }^{1}$ Gillian L. Schauer, ${ }^{2}$ Ann M. Malarcher, ${ }^{3}$ \\ Lei Zhang, ${ }^{3}$ Paul Mowery, ${ }^{4}$ and Chelsea M. Nash ${ }^{1}$ \\ ${ }^{1}$ Alere Wellbeing, Inc., 999 Third Avenue, Suite 2000, Seattle, WA 98104, USA \\ ${ }^{2}$ Carter Consulting, Inc., Contractor to Office on Smoking and Health, Centers for Disease Control and Prevention, \\ 4770 Buford Highway NE, MS F-79, Atlanta, GA 30341, USA \\ ${ }^{3}$ Office on Smoking and Health, Centers for Disease Control and Prevention, 4770 Buford Highway, NE MS F-79, Atlanta, \\ GA 30341, USA \\ ${ }^{4}$ Biostatistics, Inc., 1738 Illehaw Drive, Sarasota, FL 34239, USA
}

Correspondence should be addressed to Katrina A. Vickerman; katrina.vickerman@alere.com

Received 26 December 2014; Revised 6 May 2015; Accepted 6 May 2015

Academic Editor: Kamran Siddiqi

Copyright (C) 2015 Katrina A. Vickerman et al. This is an open access article distributed under the Creative Commons Attribution License, which permits unrestricted use, distribution, and reproduction in any medium, provided the original work is properly cited.

Objectives. To examine abstinence outcomes among tobacco users with and without a reported mental health condition (MHC) who enrolled in state tobacco quitline programs. Methods. Data were analyzed from a 7-month follow-up survey (response rate: $41 \%[3,132 / 7,459])$ of three state-funded telephone quitline programs in the United States that assessed seven self-reported MHCs at quitline registration. We examined 30-day point prevalence tobacco quit rates for callers with any MHC versus none. Data were weighted to adjust for response bias and oversampling. Multivariable logistic regression was used to examine cessation outcomes. Results. Overall, $45.8 \%$ of respondents reported $\geq 1$ MHC; $57.4 \%$ of those reporting a MHC reported $\geq 2$ MHCs. The unadjusted quit rate for callers with any MHC was lower than for callers with no MHC $(22.0 \%$ versus $31.0 \%, P<0.001)$. After adjusting for demographics, nicotine dependence, and program engagement, callers reporting $\geq 1 \mathrm{MHC}$ were less likely to be abstinent at followup (adjusted $\mathrm{OR}=0.63,95 \% \mathrm{CI}=0.51-0.78, P<0.001$ ). Conclusions. More intensive or tailored quitline programs may need to be developed among callers with MHCs as their quit rates appear to be lower than callers without MHCs.

\section{Introduction}

Individuals with mental health conditions (MHCs) are twice as likely to smoke compared to the general population [1] with smoking prevalence varying from $34 \%$ to over $60 \%$ depending on specific diagnosis [2,3]. Individuals with MHCs are also more likely to die prematurely - up to 25 years earlier on average in some populations [4] - and, similar to the general population, tobacco-related conditions such as heart disease and cancer are the leading causes of death among individuals with MHCs [4].

Smoking rates have significantly declined over the past decade among the general population [5], but rates have not declined among individuals with MHCs [6]. Although studies have shown that evidence-based treatment increases cessation among persons with MHCs [7-10], individuals with MHCs may have unique treatment needs $[2,11]$ and, based on epidemiological evidence, have a lower rate of successful cessation than those without MHCs $[1,12]$. There is growing evidence that, on average, quitting tobacco does not negatively impact long-term psychological functioning for persons with MHCs-a concern often raised by providers [13]. In fact, treatment may reduce MHC symptoms and improve functioning [14-17].

State tobacco quitlines in the United States provide evidence-based tobacco cessation treatment to more than 
400,000 smokers annually, including free phone-based cessation counseling and often access to nicotine replacement therapy (NRT) $[18,19]$. Approximately one-fourth of quitline callers meet criteria for major Depression [20] and approximately half or greater may have a $\mathrm{MHC}[21,22]$. Compared to those who try to quit with no support, quitline counseling increases the odds of quitting by $60 \%$ in the general population [11] and is a cost-effective public health intervention [23-27]. Quitlines may be a particularly valuable resource for individuals with MHCs because they reduce barriers to care (i.e., cost and transportation) [28]. Little is known about the effectiveness of quitline counseling for individuals with MHCs. Lower quit rates for callers with a $\mathrm{MHC}$ have been reported in several conference presentations $[21,22,29]$, whereas other researchers found no differences in quit rates for $\mathrm{MHC}$ and non-MHC populations [30] or for callers with and without a positive Depression screen [27]. In the two published studies examining MHCs among quitline callers, callers to the California Smokers' Helpline with major Depression were less likely to quit at two-month follow-up (19\% versus 28\%) [20], and callers to the New York State Smokers' Quitline who were heavy drinkers had lower abstinence rates at two-week follow-up compared to moderate drinkers [31]. Quit rates among quitline callers with other MHCs, such as Attention Deficit Hyperactivity Disorder, Bipolar Disorder, Generalized Anxiety Disorder, Posttraumatic Stress Disorder, and Schizophrenia, have not been published.

Accordingly, the current study examines characteristics and quit outcomes for callers to three state quitlines who reported on seven MHCs at program registration. We hypothesized that callers with MHCs would also have other characteristics that may make it more challenging for them to quit, such as higher tobacco dependence and lower socioeconomic status $[1,11,32]$ and that callers with MHCs would have lower 30 -day point prevalence quit rates at 7 month follow-up after adjusting for these factors.

\section{Methods}

2.1. Sample Selection. This observational study includes data from four evaluation samples of quitline registrants from January 2012 through May 2013: one in Maryland, one in Nebraska, and two in North Carolina (one with JanuaryJune 2012 registrants and the other with August 2012May 2013 registrants). These evaluations were conducted as part of each state's ongoing program outcome evaluation procedures. The states agreed to contribute their evaluation data and participate in this secondary data analysis. These state quitlines were selected because they asked a custom registration question to assess self-reported MHC status, offered a multiple-call phone-based cessation program, and had quitline services delivered and evaluations conducted by the same quitline vendor (Alere Wellbeing). After enrolling in the quitline program and completing their first coaching call, callers were selected for evaluation follow-up based on the following criteria. All tobacco users who had enrolled in the phone-based state quitline program in Maryland and
Nebraska were selected for evaluation (census sample), until the final three months of the Maryland evaluation, when random sampling was used due to increased quitline call volumes, and thus an influx of individuals eligible for the evaluation. Callers who were pregnant, Medicaid-insured, or reported having a MHC were oversampled using a probability sampling scheme in the North Carolina evaluations. Additional details regarding sampling methods are presented in Table 1. For all evaluations, quitline participants were eligible for inclusion if they were English or Spanish speaking, 18 years or older, and a tobacco user at enrollment, provided a valid phone number, consented to evaluation follow-up during registration, and had completed at least one coaching call. Data weighting procedures were used to accommodate population oversampling during analysis.

2.2. The Quitline Program. All three states offered a multiplecall phone-based cessation program for the duration of the study time frame, which included an initial assessment and planning call plus three to four additional coaching calls, a printed quit guide, and access to the Web Coach website, an interactive web-based cessation resource designed to complement the phone program. The quitline program is based on social cognitive theory and the United States Public Health Service Clinical Practice Guidelines [11, 33]. Calls focus on creating a quit plan, using problem solving and skills development to address cravings and triggers, leveraging social support, and using cessation medications to achieve abstinence and avoid relapse. All tobacco users who were ready to quit in the next 30 days at the time of registration were eligible for the multiple-call program. A one-call program was available for callers not ready to quit or not interested in the multiple-call program. Only a small number of callers selected the one-call program, and only 98 completed a follow-up survey (2.9\% of all completed surveys) during the study time frame. Accordingly, we have excluded those opting for the one-call program from these analyses. Free NRT was provided through the quitline to eligible callers in Maryland and North Carolina. Table 1 presents additional details regarding quitline services and NRT offerings during the study time frame.

2.3. Follow-Up Survey Administration. Evaluations were conducted in accordance with recommendations from the North American Quitline Consortium for assessing quit outcomes for state quitlines in North America [34]. Sampled participants were contacted approximately 7 months after completing their first coaching call. Participants with a valid email address who consented to being contacted via email were emailed an invitation to complete the follow-up survey online. Those who did not complete the survey after three reminder emails were then contacted by trained interviewers to complete a phone-based survey. Interviewers made at least one attempt per day to reach each participant by phone; attempts were made on up to 11 different days over approximately a 4-week period.

Across the three states, 7,646 tobacco users who enrolled in the multiple-call programs within January 2012-May 2013 
TABle 1: Quitline services for tobacco users enrolled in the Nebraska, North Carolina, or Maryland state tobacco quitlines.

\begin{tabular}{|c|c|c|c|}
\hline \multirow{2}{*}{ Services } & \multicolumn{3}{|c|}{ State quitline } \\
\hline & Nebraska & North Carolina & Maryland \\
\hline Registration dates included & $\begin{array}{l}1 \text { August 2012-31 October } \\
2012\end{array}$ & $\begin{array}{l}\text { Evaluation 1: } 1 \text { January 2012-30 June } 2012 \\
\text { Evaluation 2: } 8 \text { August 2012-31 May } 2013\end{array}$ & $\begin{array}{l}\text { 1 December 2012-31 May } \\
2013\end{array}$ \\
\hline $\begin{array}{l}7 \mathrm{M} \text { responders/number in } \\
\text { sample }\end{array}$ & $136 / 342$ & $\begin{array}{l}\text { Evaluation 1: } 827 / 1,966 \\
\text { Evaluation 2: } 753 / 1,875\end{array}$ & $1,546 / 3,463$ \\
\hline Evaluation sample selection & Census & $\begin{array}{l}\text { Evaluation 1: oversampled Medicaid } \\
\text { Evaluation 2: oversampled Medicaid, } \\
\text { pregnant, MHCs }\end{array}$ & $\begin{array}{l}\text { Census; random sampling } \\
\text { (March-May 2013) }\end{array}$ \\
\hline One-call program $^{\mathrm{a}}$ & All tobacco users & All tobacco users & All tobacco users \\
\hline $\begin{array}{l}\text { Multiple-call program } \\
\text { (Assessment and planning call } \\
\text { plus 3-4 outbound calls) }\end{array}$ & $\begin{array}{l}\text { Five-call program for } \\
\text { tobacco users ready to quit } \\
\text { in the next } 30 \text { days }\end{array}$ & $\begin{array}{l}\text { Four-call program for tobacco users } \\
\text { ready to quit in the next } 30 \text { days }\end{array}$ & $\begin{array}{l}\text { Four-call program for } \\
\text { tobacco users ready to quit } \\
\text { in the next } 30 \text { days }\end{array}$ \\
\hline Ten-call program & Pregnant tobacco users & Pregnant tobacco users & Pregnant tobacco users \\
\hline $\begin{array}{l}\text { Web Coach } \\
\text { (Interactive online complement } \\
\text { to phone coaching) }\end{array}$ & $\begin{array}{l}\text { All phone program } \\
\text { participants }\end{array}$ & All phone program participants & $\begin{array}{l}\text { All phone program } \\
\text { participants }\end{array}$ \\
\hline $\begin{array}{l}\text { Stand-alone web-based tobacco } \\
\text { cessation Program }\end{array}$ & Not offered & $\begin{array}{l}\text { For tobacco users who preferred to } \\
\text { receive only online support (starting } 1 \\
\text { January 2012) }\end{array}$ & $\begin{array}{l}\text { For tobacco users who } \\
\text { preferred to receive only } \\
\text { online support (starting } 12 \\
\text { January 2012) }\end{array}$ \\
\hline
\end{tabular}

(i) Eight-week supply of patch, lozenge, or gum to multiple-call enrollees in the following groups:

(i) Not offered

(ii) Proof of quitline enrollment and completion

Direct Mail Order (DMO) nicotine replacement therapy of a program call was a component for some Medicaid participants to receive NRT or medications through their pharmacy benefits manager (a) all (1 January 2012-20 May 2012)

(b) uninsured (starting 19 December 2012)

(c) Orange County residents (starting 13 February 2013)

(ii) Two-week (starting 13 February 2013) supply of patch, lozenge, or gum for multiple-call enrollees who were insured (expanded to 8-week supply on 22 May 2013)

(iii) Eight-week supply of patches for multiple-call enrollees with state employees' health insurance (duration of study timeframe)

Note: $7 \mathrm{M}$ = 7-month survey; MHCs = mental health conditions (conditions assessed: Attention Deficit Hyperactivity Disorder, Bipolar Disorder, Depression, Drug or Alcohol Use Disorder (or Substance Use Disorder), Generalized Anxiety Disorder, Posttraumatic Stress Disorder, and Schizophrenia).

${ }^{a}$ This study focused on callers who enrolled in a multiple-call telephone program. Individuals who selected the one-call program or the stand-alone web-based tobacco cessation program were not included. Limited 7-month evaluation data was available for these groups because a small minority selected these services and only Maryland collected follow-up data for the stand-alone web-based program during this timeframe.

were selected for evaluation; 3,262 completed the 7-month survey (response rate: $42.7 \%$ ). The final sample includes 3,132 participants (40.9\%) who responded to the question assessing MHC status during quitline registration and also provided their quit status at 7-month follow-up.

\subsection{Measures}

2.4.1. Baseline Program Registration Data. To assess MHC status at program registration, callers were asked a behavioral health question similar to one developed by a NAQC advisory forum [35]: "Do you currently have any mental health conditions, such as Attention Deficit Hyperactivity
Disorder (ADHD), Bipolar Disorder, Depression, Drug or Alcohol Use Disorder (or Substance Use Disorder; SUD), Generalized Anxiety Disorder (GAD), Posttraumatic Stress Disorder (PTSD), and Schizophrenia?" Registration agents paused briefly after each condition to allow participants to respond. We examined outcomes for callers who reported any MHC versus no MHCs. In addition, because the majority of callers with a MHC reported comorbid MHCs, we also established four mutually exclusive diagnostic groups. The groups were based on conditions that typically have the greatest impact on daily functioning and conditions that were highly comorbid in our sample, such as Depression and anxiety disorders: (Group 1) Schizophrenia or Bipolar 
Disorder; (Group 2) Depression, GAD, or PTSD but no report of Schizophrenia or Bipolar Disorder; (Group 3) SUD or ADHD but no report of the other conditions; and (Group 4) no MHC. Demographics (age, gender, education, race/ethnicity, and chronic health condition status [presence of asthma, diabetes, chronic obstructive pulmonary disease, and/or coronary artery disease]), baseline tobacco use data (type [cigarette, cigar, pipe, smokeless, and other], amount [cigarettes per day or CPD], time to first use after waking [TTFU]), and health insurance status (private, Medicare, Medicaid, and uninsured) were collected during quitline registration, per NAQC guidelines [36]. CPD and TTFU were used to create a nicotine dependence index. CPD was recorded on a continuous scale and categorized into four groups: (1) $0-10$, (2) 11-20, (3) 21-30, and (4) 31 or more. TTFU was recorded on a 4-point scale: (1) 61 or more minutes, (2) 31-60 minutes, (3) 6-30 minutes, and (4) within 5 minutes. The index is the mean of these two 4 -point scales.

2.4.2. Program Engagement. The number of coaching calls completed in the program and whether NRT was sent from the quitline were recorded and examined as indicators of program engagement.

2.4.3. 7-Month Follow-Up Survey. Tobacco cessation outcomes were assessed during the 7-month follow-up survey by asking respondents, "When did you last use tobacco, even a puff or a pinch? (Please do not include electronic cigarettes)". Respondents' self-reported last tobacco use data were used to calculate 7- and 30-day point prevalence tobacco quit rates. Participants were also asked whether they had used any cessation medications to help them quit since enrolling in the quitline (NRT patch, gum, lozenge, inhaler, nasal spray, Chantix/varenicline, Zyban/bupropion/Wellbutrin, and "other" medication).

2.5. Analyses. Data were weighted separately to the populations eligible for the evaluation for each state for nonresponse to the 7-month survey based on age, gender, race/ethnicity, insurance status, call completion, and dependence level. Data from the three states were then combined and poststratification weights were computed to adjust for oversampling in North Carolina evaluations (by pregnancy status, presence of a MHC, and Medicaid status); all variables used during response bias weighting were also included in this step. Weights were computed using a raking macro [37]. Weighted analyses were conducted in SAS 9.3 (SAS Institute Inc., Cary, NC). The purpose of these weighting procedures was to increase the generalizability of the 7-month survey results to the entire population eligible for evaluation in these states $(N=28,391)$ by adjusting the weights of individuals' 7 month survey data to ensure that respondent characteristics were similar to population characteristics on the weighting variables listed above.

Demographics, tobacco use characteristics, program engagement, and 7-month survey outcomes were examined for callers with and without reported MHCs. Multivariable logistic regression was used to assess whether callers with any MHCs were less likely to quit for 30 days or more at follow-up compared to those without MHCs, controlling for age, gender, race/ethnicity, education, insurance status, number of calls completed, use of cessation medications reported at follow-up, and state (to account for state-level differences in tobacco control environment and quitline services). Two models were estimated; the first examined any versus no MHCs, and the second examined MHC status divided into the four mutually exclusive condition groups described above (Group 1: Schizophrenia/Bipolar Disorder; Group 2: Depression/GAD/PTSD; Group 3: SUD/ADHD; Group 4: no $\mathrm{MHC}$ ). In the second model, each condition group was compared to no $\mathrm{MHC}$ and to each other.

\section{Results}

Nearly half (45.8\%) of survey respondents reported one or more MHCs at baseline including: $31.9 \%$ Depression, $21.2 \%$ GAD, 13.6\% Bipolar Disorder, 8.4\% PTSD, 7.4\% ADHD, 6.7\% SUD, and 3.6\% Schizophrenia. The majority (57.4\%) of those reporting a MHC reported two or more comorbid MHCs (26.3\% of the total sample).

Compared to those without MHCs, a higher percentage of respondents with a $\mathrm{MHC}$ were younger, female, White nonHispanic, and Medicaid-insured, had less than a high school degree, had higher tobacco dependence (based on CPD and TTFU), had a chronic health condition, and completed three or more program calls (Tables 2 and 3). A lower percentage of callers with a MHC had been mailed NRT through the quitline; however, there were no differences in self-reported use of NRT since program registration (Table 3 ). There were no differences in type of tobacco used (Table 2) or satisfaction with the quitline (Table 3).

3.1. Quit Rates for Callers with and without MHCs. At the time of the 7-month survey, the unadjusted quit rate for callers with a MHC was significantly lower than for callers without a MHC (30-day point prevalence quit rates: $22.0 \%$ $(95 \% \mathrm{CI}=19.5 \%-24.5 \%)$ versus $31.0 \%$ (95\% CI $=28.4 \%-$ $33.6 \%), P<0.001$ ) (Table 3). Multivariable logistic regression analyses confirmed that callers who reported one or more MHCs were significantly less likely to quit at follow-up (adjusted OR $=0.63,95 \% \mathrm{CI}=0.51-0.77, P<0.001$ ) (Table 4). Callers with higher baseline tobacco dependence and Medicaid insurance (compared to private) were also less likely to quit at follow-up, and callers who completed more program calls were more likely to quit.

3.2. Quit Rates by MHC Group. Unadjusted 30-day point prevalence quit rates for the mutually exclusive MHC groups were as follows: Schizophrenia or Bipolar Disorder (Group 1) [19.4\% (95\% CI = 15.4\%-23.3\%)]; Depression, GAD, or PTSD without Schizophrenia or Bipolar Disorder (Group 2) $[24.0 \%$ (95\% CI $=20.6 \%-27.5 \%)]$; SUD or ADHD without the other five conditions (Group 3) [18.3\% (95\% CI = 9.5\%-27.1\%)] (data not shown in tables). In multivariable logistic regression analyses, all three condition groups were significantly less likely to quit compared to callers with no MHC (Table 4); 
TABLE 2: Baseline characteristics among multiple-call program callers with and without self-reported MHCs in three states.

\begin{tabular}{|c|c|c|c|c|}
\hline \multirow[t]{2}{*}{ Baseline data } & $\begin{array}{c}\text { Total } \\
(N=3,132)\end{array}$ & $\begin{array}{c}\text { No MHCs } \\
(N=1,697,54.2 \%)\end{array}$ & $\begin{array}{c}\text { One or more } \\
\text { MHCs } \\
(N=1435,45.8 \%)\end{array}$ & \multirow[t]{2}{*}{$P$ value $^{\mathrm{a}}$} \\
\hline & Weighted \% & Weighted \% & Weighted \% & \\
\hline Age - mean (SD) & $46.3(13.2)$ & $47.0(13.8)$ & $45.5(12.4)$ & 0.0134 \\
\hline \multicolumn{5}{|l|}{ Gender } \\
\hline Male & 36.6 & 39.8 & 32.7 & \multirow{2}{*}{0.0005} \\
\hline Female & 63.4 & 60.2 & 67.3 & \\
\hline \multicolumn{5}{|l|}{ Education } \\
\hline Less than high school & 21.0 & 18.4 & 24.1 & \multirow{5}{*}{0.0007} \\
\hline GED & 6.4 & 5.7 & 7.1 & \\
\hline High school degree & 28.7 & 31.4 & 25.6 & \\
\hline Some college/trade school & 27.5 & 27.2 & 27.9 & \\
\hline College/trade school degree & 16.4 & 17.4 & 15.2 & \\
\hline \multicolumn{5}{|l|}{ Race/ethnicity } \\
\hline White, non-Hispanic & 59.7 & 52.0 & 68.9 & \multirow{4}{*}{$<0.0001$} \\
\hline Black, non-Hispanic & 33.2 & 39.9 & 25.1 & \\
\hline Hispanic & 2.4 & 2.9 & 1.9 & \\
\hline Other, non-Hispanic & 4.7 & 5.2 & 4.1 & \\
\hline \multicolumn{5}{|l|}{ Insurance status } \\
\hline Medicaid & 22.1 & 16.5 & 28.8 & \multirow{4}{*}{$<0.0001$} \\
\hline Uninsured & 39.7 & 42.2 & 36.7 & \\
\hline Private & 23.9 & 29.0 & 17.8 & \\
\hline Medicare & 14.3 & 12.2 & 16.7 & \\
\hline \multicolumn{5}{|l|}{ Tobacco type ${ }^{b}$} \\
\hline Cigarette & 97.6 & 97.0 & 98.1 & 0.0978 \\
\hline Cigar & 2.7 & 2.6 & 2.9 & 0.7038 \\
\hline Pipe & 0.3 & 0.4 & 0.3 & 0.7091 \\
\hline Smokeless & 2.0 & 2.2 & 1.7 & 0.4111 \\
\hline Other & 0.8 & 0.5 & 1.2 & 0.1066 \\
\hline CPD - mean (SD) & $18.6(11.3)$ & $17.7(10.5)$ & $19.8(12.0)$ & $<0.0001$ \\
\hline $0-10$ & 30.5 & 32.8 & 27.9 & \multirow{4}{*}{0.0003} \\
\hline $11-20$ & 46.7 & 47.6 & 45.6 & \\
\hline $21-30$ & 12.7 & 11.9 & 13.6 & \\
\hline $31+$ & 10.1 & 7.8 & 12.9 & \\
\hline \multicolumn{5}{|l|}{ Time to first use } \\
\hline$<5 \min$ & 52.9 & 49.0 & 57.6 & \multirow{4}{*}{0.0003} \\
\hline $6-30 \mathrm{~min}$ & 28.7 & 30.3 & 26.9 & \\
\hline $31-60 \mathrm{~min}$ & 9.0 & 9.8 & 8.1 & \\
\hline $60 \min +$ & 9.3 & 10.9 & 7.4 & \\
\hline Dependence index ${ }^{c}$ & $2.6(0.8)$ & $2.6(0.8)$ & $2.7(0.8)$ & $<0.0001$ \\
\hline Below median (1-2.5) & 52.3 & 55.9 & 47.9 & \multirow{2}{*}{0.0001} \\
\hline Above median (3-4) & 47.7 & 44.1 & 52.1 & \\
\hline \multicolumn{5}{|l|}{ Chronic health conditions } \\
\hline Any of 4 conditions & 45.9 & 38.3 & 54.9 & $<0.0001$ \\
\hline Asthma & 18.4 & 11.4 & 26.5 & $<0.0001$ \\
\hline Diabetes & 14.9 & 12.6 & 17.5 & 0.0004 \\
\hline COPD & 19.2 & 14.5 & 24.7 & $<0.0001$ \\
\hline CAD & 9.2 & 8.2 & 10.4 & 0.0481 \\
\hline
\end{tabular}

Note: $\mathrm{MHCs}=$ mental health conditions (conditions assessed: Attention Deficit Hyperactivity Disorder, Bipolar Disorder, Depression, Drug or Alcohol Use Disorder (or Substance Use Disorder), Generalized Anxiety Disorder, Posttraumatic Stress Disorder, and Schizophrenia); GED = General Education Development; $\mathrm{CPD}=$ cigarettes per day; time to first use = time to first tobacco use after waking; $\mathrm{COPD}=$ chronic obstructive pulmonary disease; $\mathrm{CAD}=$ coronary artery disease.

${ }^{a} P$ values computed using proc surveylogistic for categorical variables and proc surveyreg for continuous variables. $P$ values tested for significant differences in baseline variable proportions or mean values for callers who reported no MHCs versus 1 or more MHCs; a cutoff of $P<0.05$ was used for statistical significance. Missing data are excluded for each variable.

${ }^{\mathrm{b}}$ These are not mutually exclusive categories. Participants could choose multiple tobacco products, if appropriate.

${ }^{c}$ Four-point scale index to represent tobacco dependence level based on cigarettes per day and time to first tobacco use after waking. Higher scores on the index represent a higher level of tobacco dependence. 
TABLE 3: Program engagement and 7-month survey outcomes among multiple-call program callers with and without self-reported MHCs in three states.

\begin{tabular}{|c|c|c|c|c|}
\hline \multirow[t]{2}{*}{$\begin{array}{l}\text { Program engagement and 7-month survey } \\
\text { responses }\end{array}$} & $\begin{array}{c}\text { Total } \\
(N=3,132)\end{array}$ & $\begin{array}{c}\text { No MHCs } \\
(N=1,697,54.2 \%)\end{array}$ & $\begin{array}{c}\text { One or more } \\
\text { MHCs } \\
(N=1435,45.8 \%)\end{array}$ & \multirow[t]{2}{*}{$P$ value $^{\mathrm{a}}$} \\
\hline & Weighted \% & Weighted \% & Weighted \% & \\
\hline \multicolumn{5}{|c|}{ Program engagement } \\
\hline Calls completed - mean (SD) & $1.8(1.0)$ & $1.8(1.0)$ & $1.9(1.1)$ & 0.0020 \\
\hline $1-2$ & 80.1 & 82.3 & 77.5 & \multirow{2}{*}{0.0008} \\
\hline $3+$ & 19.9 & 17.7 & 22.5 & \\
\hline Received NRT from quitline & 74.6 & 79.2 & 69.1 & $<0.0001$ \\
\hline \multicolumn{5}{|c|}{ Seven-month survey responses } \\
\hline $\begin{array}{l}\text { Used cessation medication to help quit since } \\
\text { enrollment }\end{array}$ & 74.0 & 73.7 & 74.4 & 0.7196 \\
\hline Satisfied with quitline program & 92.9 & 93.1 & 92.6 & 0.6376 \\
\hline Quit 7 days & 31.9 & 35.5 & 27.6 & $<0.0001$ \\
\hline Quit 30 days & 26.9 & 31.0 & 22.0 & $<0.0001$ \\
\hline
\end{tabular}

Note: MHCs = mental health conditions (conditions assessed: Attention Deficit Hyperactivity Disorder, Bipolar Disorder, Depression, Drug or Alcohol Use Disorder (or Substance Use Disorder), Generalized Anxiety Disorder, Posttraumatic Stress Disorder, and Schizophrenia).

${ }^{a} P$ values computed using proc surveylogistic for categorical variables and proc surveyreg for continuous variables. $P$ values tested for significant differences in program engagement and 7-month survey variable proportions or mean values for callers who reported no MHCs versus 1 or more MHCs; a cutoff of $P<0.05$ was used for statistical significance. Missing data are excluded for each variable.

the three MHC groups did not significantly differ in likelihood of being quit [Group 1 versus 2: adjusted OR $=0.76(95 \%$ $\mathrm{CI}=0.54-1.06), P=0.11$; Group 1 versus 3: adjusted $\mathrm{OR}=$ 1.21 (95\% CI $=0.58-2.51), P=0.61$; Group 2 versus 3: adjusted $\mathrm{OR}=1.60(95 \% \mathrm{CI}=0.79-3.22), P=0.19]$.

\section{Discussion}

Among callers to three state quitline multiple-call programs, nearly half (46\%) reported one of seven current MHCs, and callers reporting a MHC were significantly less likely to quit at 7-month follow-up compared to callers without a MHC. After controlling for demographics, baseline tobacco dependence, program utilization characteristics, and state, callers with a MHC had 0.6 times lower adjusted odds of being quit compared to callers without a MHC. These findings may seem to suggest that callers with MHCs are not benefiting from quitline services; however, less than $10 \%$ of people in the general population who use no support or minimal selfhelp successfully quit smoking [11]. Epidemiological research indicates that odds of successfully quitting may be even lower for individuals with MHCs $[1,12]$. Because this was an observational study, we could not determine what the relative likelihood of quitting without assistance would have been for those with and without a MHC, so we could not determine how much the quitline intervention increased the odds of success, and whether this differed for callers with and without a MHC.

Quitline callers with MHCs also have other characteristics that have been shown in previous research to make quitting harder, including higher tobacco dependence, lower education and socioeconomic status, and having other chronic health conditions $[1,11,32]$. Given that callers with MHCs are more likely to have sociodemographic characteristics associated with higher tobacco use and greater difficulty quitting, improving treatment for callers with MHCs may also help other priority populations disproportionately impacted by tobacco use. Assessing mutually exclusive MHC groups, we found that all three condition groups differed from callers with no MHCs in multivariable models but did not differ from one another. Together with the finding that callers with any MHCs were less likely to quit than those with none, this suggests that any report of a MHC may predict increased difficulty in quitting, even though the population of individuals with any MHC is likely a heterogeneous group in terms of symptoms, stressors, and daily functioning.

Despite having characteristics that can hinder quitting, our data suggest that callers with MHCs are engaged in the quitting process. For example, callers with MHCs were more likely than those without MHCs to complete three or more program calls, which has been associated with greater quit success $[24,32,38]$. Findings indicating greater treatment engagement among those with MHCs could be due to an increased need for support and/or high motivation to change. More research is needed to assess why quitline program engagement is higher in this population.

Callers with and without MHCs were equally likely to report having used cessation medications during their quit attempt; however, fewer callers with MHCs were sent medications through the quitlines, which may have been due to NRT contraindication guidelines, eligibility criteria for the specific states, or interest in using medications not covered by the quitlines (e.g., varenicline and bupropion). It is encouraging that callers with MHCs obtained cessation medications from other sources, particularly given that callers with MHCs are more likely to have higher tobacco dependence $[1,20]$ 
TABLE 4: Multivariable models of the relationship of 30-Day tobacco abstinence and MHC status, by any conditions versus none (Model 1) and by condition group versus none (Model 2) in three states.

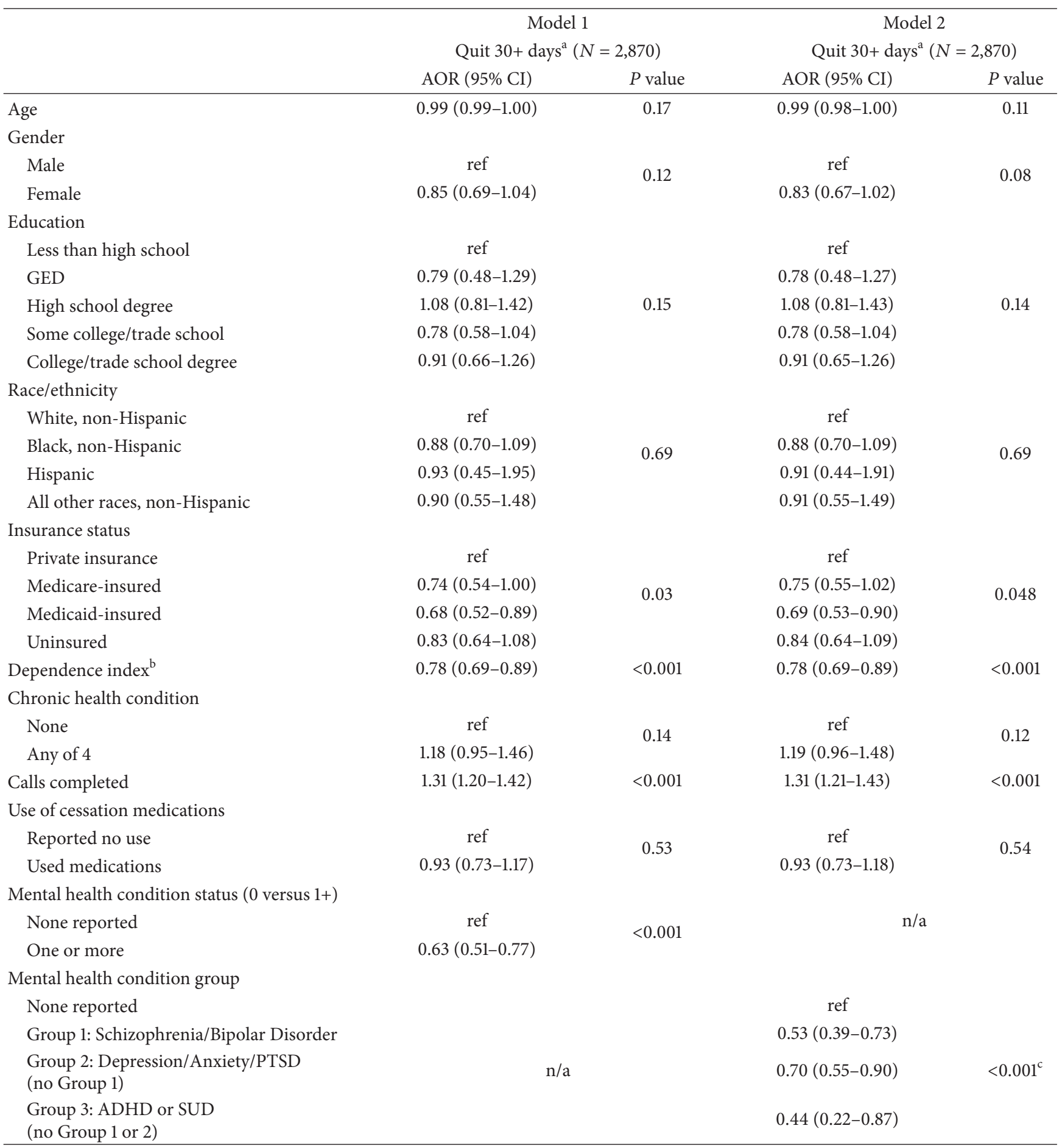

Note: $N$ = total sample size; AOR = Adjusted Odds Ratio; $\mathrm{CI}$ = Confidence Interval; ref = reference group.

${ }^{a} 30$-day point prevalence abstinence at 7-month survey.

${ }^{b}$ Four-point scale index to represent tobacco dependence level based on cigarettes per day and time to first tobacco use after waking. Higher scores on the index represent a higher level of tobacco dependence.

${ }^{c^{c}}$ The three MHC groups did not significantly differ in likelihood of being quit (Group 1 versus 2: adjusted OR $=0.76(95 \% \mathrm{CI}=0.54-1.06)$, $P=0.11$; Group 1 versus 3: adjusted $\mathrm{OR}=1.21$ (95\% $\mathrm{CI}=0.58-2.51), P=0.61$; Group 2 versus 3: adjusted $\mathrm{OR}=1.60(95 \% \mathrm{CI}=0.79-3.22), P=0.19)$.

Notes: models also included state as a fixed effect. Callers with missing data on one or more model variables were excluded from the model. $P$ values indicate whether variables were a significant predictor of quit status in the multivariable models; a cutoff of $P<0.05$ was used for statistical significance. 
and may need more intensive medication support than the general population $[11,28,39]$. Since barriers such as copays and prior authorizations can negatively impact access to cessation medication and quitting success [40-42], provision of free NRT directly through quitlines could further improve medication use for all callers. Reported medication use was not a significant predictor of quit status at 7 months in the multivariable model, which is not an unusual finding in quitline observational studies where participants self-select whether to use cessation medications [32]; this should not be interpreted as medications being unimportant in callers' quitting process.

More knowledge is needed about mechanisms of connection between smoking addiction and MHCs, which may be environmental or social (e.g., tobacco norms and smoking exposure among peers and in treatment facilities), result from common genetic predispositions, brain functioning or other risk factors, or stem from behavior associations or symptom management habits (e.g., alcohol use as a trigger for smoking and vice versa and symptom self-medication) [5, 43, 44]. Reasons for comorbidity between tobacco addiction and MHCs also may differ for specific disorders. Determining the best method of assessing and identifying callers with MHCs will aid in and inform research on whether tailored treatments improve outcomes for callers with MHCs and how best to tailor treatment.

Experts have put forth recommendations for tailoring treatment for people with MHCs. These include provision of more intensive counseling, higher doses of NRT or combination therapy, and cessation medications that also target mood (i.e., varenicline and bupropion) $[2,11,15,28,39]$. Care comanagement with mental health providers has been suggested, particularly with psychiatric medication prescribers. Since nicotine impacts how some common psychiatric medications are metabolized, medications may need adjustment during and after quitting $[11,28,45,46]$. Treatment providers may also need to address tobacco users' concerns about weight gain, which could be complicated by medication side effects and higher rates of inactivity, focus on beliefs about self-medication with tobacco and alternative coping strategies, and use concrete smaller goals for individuals with lower cognitive functioning $[2,15,28,39]$. Finally, changes in psychiatric symptoms should be monitored during quitting $[2,17,28]$. Strategies such as reducing to quit, pairing tobacco quitline treatment with a brief alcohol intervention, or combining quitline and community treatment may also warrant additional research $[2,31]$.

More research is needed to test which recommendations yield improvements in quit outcomes, particularly in quitline settings. Several quitline studies provide relevant findings to inform future research. Outcomes were similar for tobacco users with and without a psychiatric history (identified via chart review) who received 12 weeks of varenicline and phone and/or web-based behavioral treatment [8]. Given these findings, future research should examine whether varenicline is particularly effective for callers with MHCs. Second, a prospective study of callers to the Victorian Quitline in
Australia provides some support for a quitline-doctor comanagement model; $83 \%$ of callers who self-disclosed doctordiagnosed Depression believed it would be beneficial to involve their doctor in their quit attempt, and those receiving comanagement were more likely to make a quit attempt [46]. Finally, a promising randomized controlled trial in the Dutch National Quitline examined whether a mood management component integrated into standard quitline treatment improved outcomes over the standard program for callers with past major Depression; they found the additional sessions and content increased prolonged abstinence rates at 6 and 12 months but did not impact a recurrence of depressive symptoms, and differences in 7-day point prevalence abstinence rates were not significant [47].

4.1. Limitations. A number of limitations should be considered when interpreting these findings. First, MHCs in this study were assessed by asking callers whether they currently had one of seven MHCs. This assessment method may not have captured individuals with undiagnosed MHCs, disorders not assessed (e.g., anxiety disorders other than GAD or PTSD), subclinical symptoms, or a diagnosis they preferred not to report. Failure to capture all individuals with clinical or subclinical MHCs, or the potential for false positives from people who self-reported "yes" to an MHC but may not have been screened positive for symptoms, may have impacted results. For example, previous research suggests that individuals with subclinical levels of MHCs are more likely to smoke [48], and subclinical levels may reduce the likelihood of quit success [49]. While rates of any reported MHCs were higher in this sample (46\%) compared to those estimated in the general population of smokers in the United States (30\%, excluding SUD [1]), the high rate of MHCs is not surprising since many state quitlines target underserved populations, and rates of MHCs are higher in smokers $[1,6]$. The assessment method used may still underidentify MHCs in our sample. For example, SUD appears likely to be underreported by participants (6.7\% in this study versus $23 \%$ of callers when amount of drinking was assessed in a New York State Smokers' Quitline study [31]). When developing the questions used in this study, an expert quitline workgroup convened by NAQC considered assessment options while weighing time and other treatment considerations [2]. However, as the workgroup noted, more research is needed to identify the most effective and efficient assessment approach for the more than 400,000 annual tobacco quitline callers.

Second, many states offer a one-call program instead of or in addition to a multiple-call program. We did not have sufficient samples to examine outcomes for one-call program enrollees with and without a MHC. Based on recommendations for more intensive treatment $[2,28]$, we expect onecall programs may be less effective for callers with MHCs. Third, our study focused on only three states, which may limit the generalizability of findings. However, our findings concur with unpublished data presented at conferences for more than seven other states $[21,22,29]$. Fourth, the 7month survey response rate was $41 \%$. This is in line with state 
quitline evaluation survey response rates reported elsewhere $[18,32]$ but again may impact the generalizability of findings. All analyses included weights to adjust for survey response bias to improve the representativeness of results. Finally, our outcome measure was self-reported abstinence from tobacco for 30 or more days at the time of the 7-month survey; we did not examine prolonged abstinence and did not have access to biochemically verified quit status (i.e., cotinine or carbon monoxide data). We used this outcome metric because it is the standard for evaluating quitline outcomes in North America [34]. Furthermore, the Society for Research on Nicotine and Tobacco Subcommittee on Biochemical Verification has recommended that biochemical verification of abstinence is not necessary in large-scale studies with no face-to-face contact where data collection is done by mail, telephone, or internet [50].

\section{Conclusion}

More research is needed to address the best approach to treatment for quitline callers with MHCs, to determine what information should be assessed to provide the best care to quitline callers with MHCs (e.g., diagnoses, symptoms, and current medications), and to understand relapse profiles, reasons, and timing for this population. Given that half of quitline callers report a MHC and these callers had significantly lower quit rates, development and testing of more intensive or tailored programs to improve outcomes are warranted. Mental health and tobacco control communities should continue to develop partnerships to address this health disparity.

\section{Disclosure}

Katrina A. Vickerman and Chelsea M. Nash declare employment at Alere Wellbeing, the provider of quitline services in this study.

\section{Disclaimer}

The findings and conclusions in this report are those of the authors and do not necessarily represent the official position of the Centers for Disease Control and Prevention.

\section{Conflict of Interests}

The authors declare no other conflict of interests regarding the publication of this paper.

\section{Acknowledgments}

This research was funded by the Centers for Disease Control and Prevention. The Maryland, Nebraska, and North Carolina state quitlines funded the collection of the 7-month survey data analyzed in this study as part of their quitline program evaluation plan. The authors thank Drs. Tim McAfee and Susan Zbikowski for their review and feedback on this paper and for input on the study design. The authors also acknowledge the three state quitlines that participated in the study.

\section{References}

[1] Centers for Disease Control and Prevention (CDC), "Vital signs: current cigarette smoking among adults aged $\geq 18$ years with mental illness-United States, 2009-2011," Morbidity and Mortality Weekly Report, vol. 62, no. 5, pp. 81-87, 2013.

[2] C. Morris, "Do quitlines have a role in serving the tobacco cessation needs of persons with mental illnesses and substance abuse disorders?" A Background Report 9-13-0014, University of Colorado, Boulder, Colo, USA, 2010.

[3] A. K. McClave, L. R. McKnight-Eily, S. P. Davis, and S. R. Dube, "Smoking characteristics of adults with selected lifetime mental illnesses: results from the 2007 national health interview survey," American Journal of Public Health, vol. 100, no. 12, pp. 2464-2472, 2010.

[4] C. W. Colton and R. W. Manderscheid, "Congruencies in increased mortality rates, years of potential life lost, and causes of death among public mental health clients in eight states," Preventing Chronic Disease, vol. 3, article A42, 2006.

[5] US Department of Health and Human Services, The Health Consequences of Smoking: 50 Years of Progress: A Report of the Surgeon General, US Department of Health and Human Services, Centers for Disease Control and Prevention, National Center for Chronic Disease Prevention and Health Promotion, Office on Smoking and Health, Atlanta, Ga, USA, 2014.

[6] B. Lê Cook, G. F. Wayne, E. N. Kafali, Z. Liu, C. Shu, and M. Flores, "Trends in smoking among adults with mental illness and association between mental health treatment and smoking cessation," Journal of the American Medical Association, vol. 311, no. 2, pp. 172-182, 2014.

[7] M. McFall, A. J. Saxon, C. E. Thompson et al., "Improving the rates of quitting smoking for veterans with posttraumatic stress disorder," American Journal of Psychiatry, vol. 162, no. 7, pp. 13111319, 2005.

[8] J. B. McClure, G. E. Swan, S. L. Catz et al., "Smoking outcome by psychiatric history after behavioral and varenicline treatment," Journal of Substance Abuse Treatment, vol. 38, no. 4, pp. 394402, 2010.

[9] S. M. Hall, J. Y. Tsoh, J. J. Prochaska et al., "Treatment for cigarette smoking among depressed mental health outpatients: a randomized clinical trial," American Journal of Public Health, vol. 96, no. 10, pp. 1808-1814, 2006.

[10] N. El-Guehaly, J. Cathcart, S. Currie, D. Brown, and S. Gloster, "Smoking cessation approaches for persons with mental illness or addictive disorders," Psychiatric Services, vol. 53, no. 9, pp. 1166-1170, 2002.

[11] M. Fiore, Treating Tobacco Use and Dependence: 2008 Update Clinical Practice Guideline, Diane Publishing, Darby, Pa, USA, 2008.

[12] K. Lasser, J. W. Boyd, S. Woolhandler, D. U. Himmelstein, D. McCormick, and D. H. Bor, "Smoking and mental illness: a population-based prevalence study," Journal of the American Medical Association, vol. 284, no. 20, pp. 2606-2610, 2000.

[13] J. J. Prochaska, "Failure to treat tobacco use in mental health and addiction treatment settings: a form of harm reduction?" Drug and Alcohol Dependence, vol. 110, no. 3, pp. 177-182, 2010.

[14] J. J. Prochaska, K. Delucchi, and S. M. Hall, "A meta-analysis of smoking cessation interventions with individuals in substance 
abuse treatment or recovery," Journal of Consulting and Clinical Psychology, vol. 72, no. 6, pp. 1144-1156, 2004.

[15] J. J. Prochaska, R. S. Reyes, S. A. Schroeder, A. S. Daniels, A. Doederlein, and B. Bergeson, "An online survey of tobacco use, intentions to quit, and cessation strategies among people living with bipolar disorder," Bipolar Disorders, vol. 13, no. 5-6, pp. 466-473, 2011.

[16] C. D. Morris, J. A. Waxmonsky, M. G. May, D. G. Tinkelman, M. Dickinson, and A. A. Giese, "Smoking reduction for persons with mental illnesses: 6-Month results from community-based interventions," Community Mental Health Journal, vol. 47, no. 6, pp. 694-702, 2011.

[17] M. S. McDermott, T. M. Marteau, G. J. Hollands, M. Hankins, and P. Aveyard, "Change in anxiety following successful and unsuccessful attempts at smoking cessation: cohort study," British Journal of Psychiatry, vol. 202, no. 1, pp. 62-67, 2013.

[18] North American Quitline Consortium (NAQC), Results from the 2012 NAQC Annual Survey of Quitlines (Webinar Presentation), North American Quitline Consortium (NAQC), Phoenix, Ariz, USA, 2013.

[19] Centers for Disease Control and Prevention, "State Tobacco Activities Tracking and Evaluation (STATE) System," http:// www.cdc.gov/tobacco/statesystem.

[20] K. K. Hebert, S. E. Cummins, S. Hernndez, G. J. Tedeschi, and S.-H. Zhu, "Current major depression among smokers using a state quitline," American Journal of Preventive Medicine, vol. 40, no. 1, pp. 47-53, 2011.

[21] A. Lukowski, D. Tinkelman, C. Morris, and S. Young, "Six-state quitline outcomes for smokers with psychiatric conditions and limitations," in Proceedings of the 20th Annual Meeting of the Society for Research on Nicotine and Tobacco (SRNT '14), Seattle, Wash, USA, February 2014.

[22] L. Beebe, S. Martinez, and S. Gillaspy, "Quitline utilization and outcomes among tobacco users with mental health and substance abuse disorders," in Proceedings of the 20th Annual Meeting of the Society for Research on Nicotine and Tobacco (SRNT '14), Seattle, Wash, USA, February 2014.

[23] C. T. Orleans, V. J. Schoenbach, E. H. Wagner et al., "Selfhelp quit smoking interventions: effects of self-help materials, social support instructions, and telephone counseling," Journal of Consulting and Clinical Psychology, vol. 59, no. 3, pp. 439-448, 1991.

[24] S. M. Zbikowski, L. M. Jack, J. B. McClure et al., "Utilization of services in a randomized trial testing phone- and web-based interventions for smoking cessation," Nicotine and Tobacco Research, vol. 13, no. 5, pp. 319-327, 2011.

[25] T. M. Bush, T. McAfee, M. Deprey et al., "The impact of a free nicotine patch starter kit on quit rates in a state quit line," Nicotine and Tobacco Research, vol. 10, no. 9, pp. 1511-1516, 2008.

[26] J. F. Hollis, T. A. McAfee, J. L. Fellows, S. M. Zbikowski, M. Stark, and K. Riedlinger, "The effectiveness and cost effectiveness of telephone counselling and the nicotine patch in a state tobacco quitline," Tobacco Control, vol. 16, no. 1, pp. i53-i59, 2007.

[27] T. McAfee, S. Tutty, K. Wassum, and A. Roberts, "Mental health and quitlines: perspectives and a pilot study of a functional assessment. Part of a moderated session: do people with mental illnesses and substance use disorders have equal access to quitlines and other smoking cessation services? Should they?" in Proceedings of the Conference of the North American Quitline Consortium (NAQC '09), Phoenix, Ariz, USA, June 2009.

[28] C. D. Morris, G. J. Tedeschi, J. A. Waxmonsky, M. May, and A. A. Giese, "Tobacco quitlines and persons with mental illnesses: perspective, practice, and direction," Journal of the American Psychiatric Nurses Association, vol. 15, no. 1, pp. 32-40, 2009.

[29] B. L. Kreinbring and L. Dale, "A quitline experience providing counseling to callers with mental illness," in Proceedings of the National Conference on Tobacco or Health (NCTOH '07), Minneapolis, Minn, USA, October 2007.

[30] M. Hrywna, C. Delnevo, J. Williams, U. Vorbach, G. Ernst, and D. Gundersen, "Use of quitline by smokers with mental illness," in Proceedings of the National Conference on Tobacco or Health (NCTOH '07), Minneapolis, Minn, USA, October 2007.

[31] B. A. Toll, K. M. Cummings, S. S. O’Malley et al., "Tobacco quitlines need to assess and intervene with callers' hazardous drinking," Alcoholism: Clinical and Experimental Research, vol. 36, no. 9, pp. 1653-1658, 2012.

[32] T. Bush, S. M. Zbikowski, L. Mahoney, M. Deprey, P. Mowery, and B. Cerutti, "State quitlines and cessation patterns among adults with selected chronic diseases in 15 states, 2005-2008.," Preventing chronic disease, vol. 9, article E163, 2012.

[33] A. Bandura, "Social cognitive theory," in Annals of Child Development, R. Vasta, Ed., vol. 6 of Six Theories of Child Development, pp. 1-60, JAI Press, Greenwich, Conn, USA, 1989.

[34] North American Quitline Consortium (NAQC), Measuring Quit Rates, Quality Improvement Initiative, Phoenix, Ariz, USA, 2009.

[35] North American Quitline Consortium (NAQC), MDS Standard Optional Questions: Behavioral Health Screening, NAQC, 2011.

[36] North American Quitline Consortium (NAQC), MDS Intake Questions, North American Quitline Consortium, 2012.

[37] D. Izrael, M. Battaglia, and M. Frankel, "Extreme survey weight adjustment as a component of sample balancing (aka raking) (paper 247)," in Proceedings of the SAS Global Forum 2009 Conference, Washington, DC, USA, March 2009, http:// support.sas.com/resources/papers/proceedings09/247-2009.pdf.

[38] L. F. Stead, R. Perera, and T. Lancaster, "A systematic review of interventions for smokers who contact quitlines," Tobacco Control, vol. 16, supplement 1, pp. i3-i8, 2007.

[39] S. A. Schroeder and C. D. Morris, "Confronting a neglected epidemic: tobacco cessation for persons with mental illnesses and substance abuse problems," Annual Review of Public Health, vol. 31, pp. 297-314, 2010.

[40] J. L. Cox and J. P. McKenna, "Nicotine gum: does providing it free in a smoking cessation program alter success rates?" Journal of Family Practice, vol. 31, no. 3, pp. 278-280, 1990.

[41] J. R. Hughes, W. C. Wadland, J. W. Fenwick, J. Lewis, and W. K. Bickel, "Effect of cost on the self-administration and efficacy of nicotine gum: a preliminary study," Preventive Medicine, vol. 20, no. 4, pp. 486-496, 1991.

[42] S. J. Curry, L. C. Grothaus, T. Mcafee, and C. Pabiniak, "Use and cost effectiveness of smoking-cessation services under four insurance plans in a health maintenance organization," The New England Journal of Medicine, vol. 339, no. 10, pp. 673-679, 1998.

[43] J. L. Heffner, J. R. Strawn, M. P. Delbello, S. M. Strakowski, and R. M. Anthenelli, "The co-occurrence of cigarette smoking and bipolar disorder: phenomenology and treatment considerations," Bipolar Disorders, vol. 13, no. 5-6, pp. 439-453, 2011.

[44] F. J. McClernon and S. H. Kollins, "ADHD and smoking: from genes to brain to behavior," Annals of the New York Academy of Sciences, vol. 1141, pp. 131-147, 2008.

[45] M. Ashir and L. Petterson, "Smoking bans and clozapine levels," Advances in Psychiatric Treatment, vol. 14, no. 5, pp. 398-399, 2008. 
[46] C. J. Segan, R. Borland, K. A. Wilhelm et al., "Helping smokers with depression to quit smoking: collaborative care with Quitline," Medical Journal of Australia, vol. 195, no. 3, pp. S7-S11, 2011.

[47] R. M. van der Meer, M. C. Willemsen, F. Smit, P. Cuijpers, and G. M. Schippers, "Effectiveness of a mood management component as an adjunct to a telephone counselling smoking cessation intervention for smokers with a past major depression: a pragmatic randomized controlled trial," Addiction, vol. 105, no. 11, pp. 1991-1999, 2010.

[48] K. Landolt, V. Ajdacic-Gross, J. Angst et al., "Smoking and psychiatric disorders: have subthreshold disorders been overlooked?" Nicotine and Tobacco Research, vol. 12, no. 5, pp. 516520, 2010.

[49] R. Niaura, D. M. Britt, W. G. Shadel, M. Goldstein, D. Abrams, and R. Brown, "Symptoms of depression and survival experience among three samples of smokers trying to quit," Psychology of Addictive Behaviors, vol. 15, no. 1, pp. 13-17, 2001.

[50] SRNT Subcommittee on Biochemical Verification, "Biochemical verification of tobacco use and cessation," Nicotine \& Tobacco Research, vol. 4, pp. 149-159, 2002. 


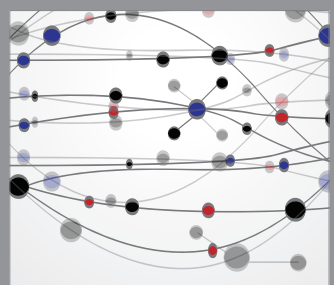

The Scientific World Journal
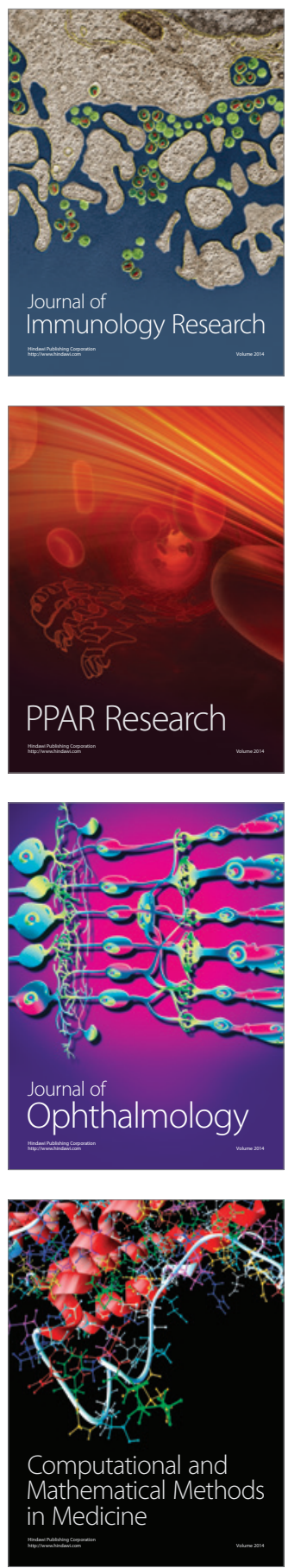

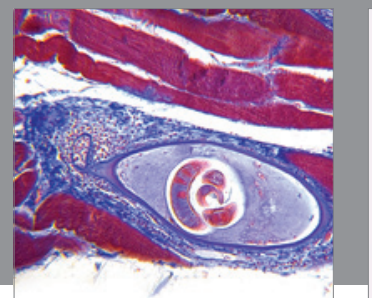

Gastroenterology

Research and Practice
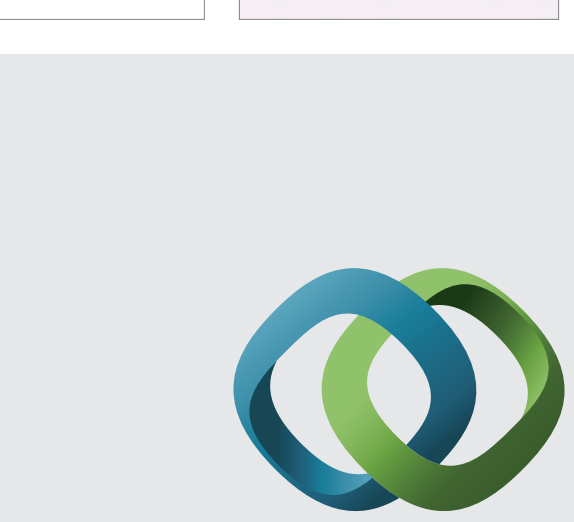

\section{Hindawi}

Submit your manuscripts at

http://www.hindawi.com
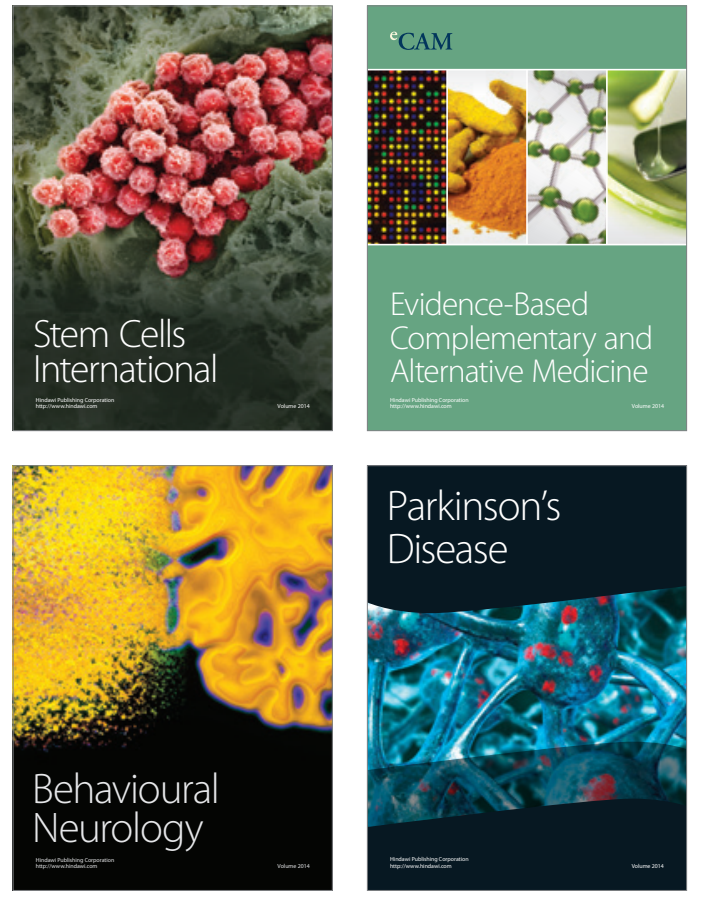
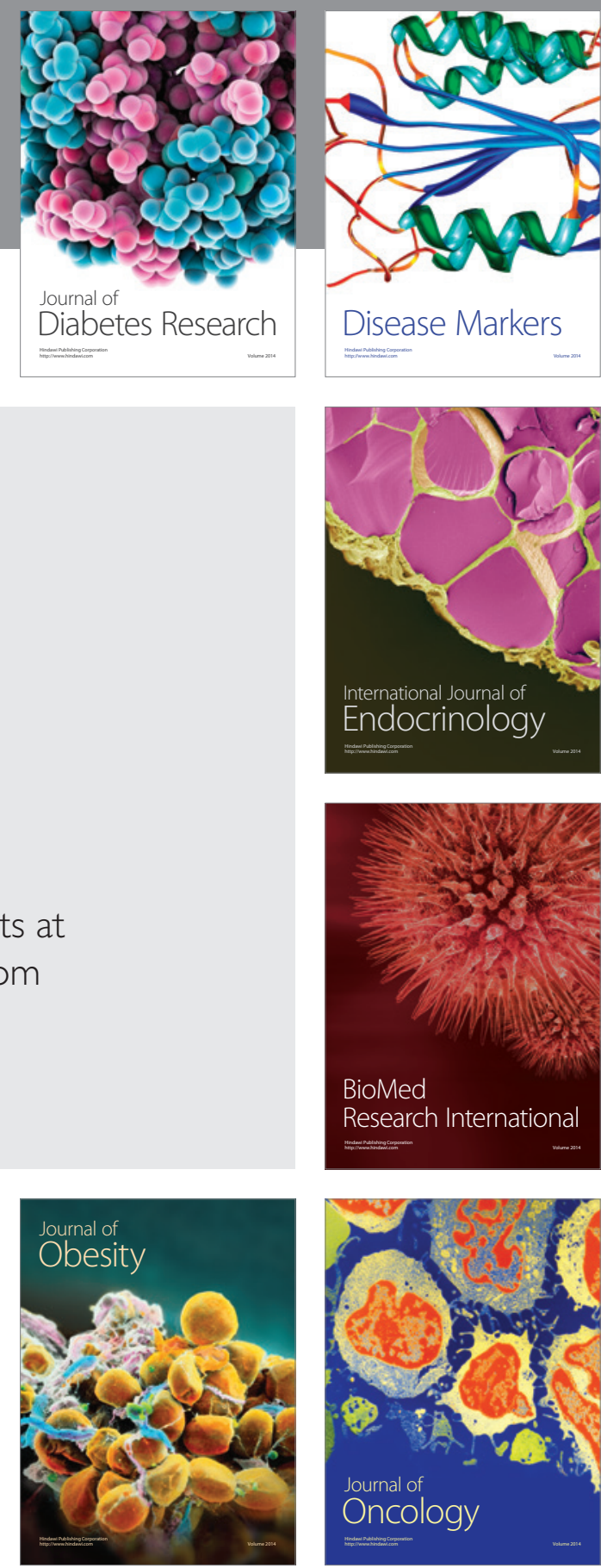

Disease Markers
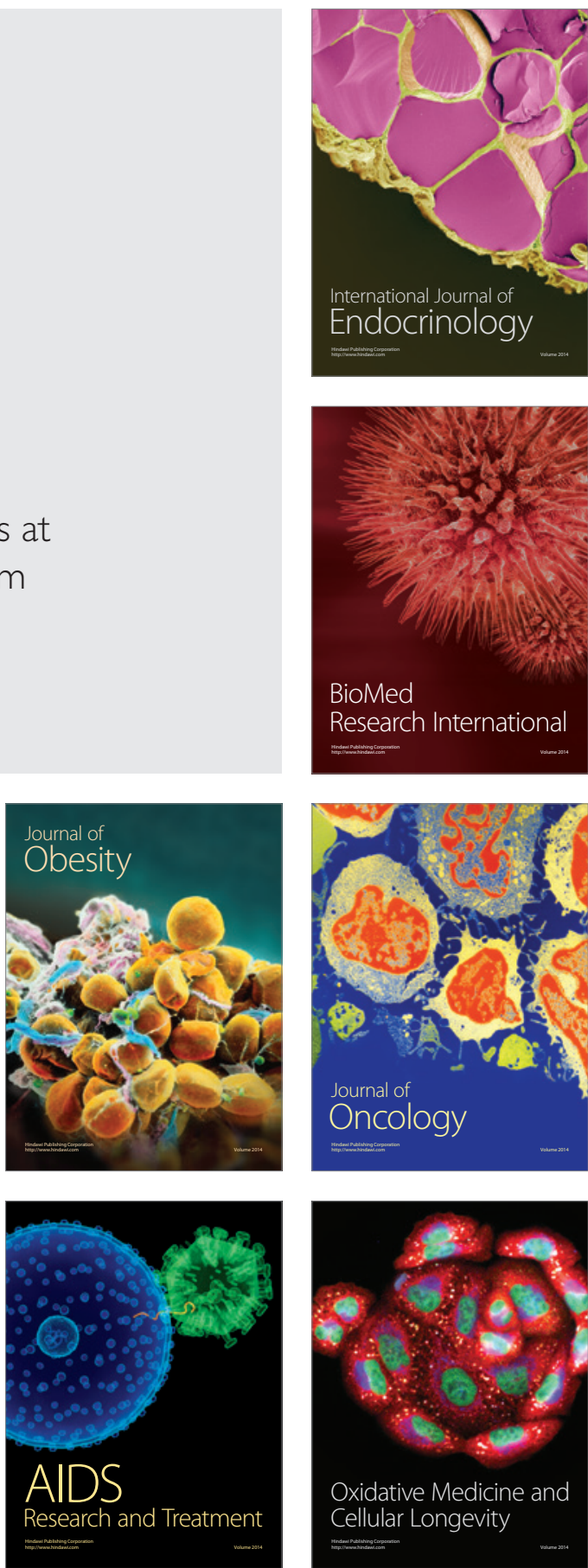